\title{
A Theoretical Survey on Patch Antenna for Wideband Communication
}

\author{
Kali Krishna Giri ${ }^{1 *}$, Raj Kumar Singh ${ }^{2}$, Kumari Mamata ${ }^{3}$, Ajeet Kumar Shrivastava ${ }^{4}$ \\ ${ }^{I}$ Research Scholar, University Department of Physics, Ranchi University, Ranchi, India \\ ${ }^{2}$ Assistant Professor, University Department of Physics, Ranchi University, Ranchi, India \\ ${ }^{3}$ Department of Applied Physics, Cambridge Institute Technology, Ranchi University, Ranchi, India \\ ${ }^{4}$ Professor, Raman University, Bilaspur, India \\ *Corresponding author: girikalikrishna@gmail.com
}

\begin{abstract}
Modern communication system is based on wideband communication. A wideband antenna is designed in such a way that it will receive a wide range of frequencies. Microwave frequency spectrum is classified as ranging from $1 \mathrm{GHz}$ to $100 \mathrm{GHz}$ and this range is divided into a number of frequency bands. These bands are defined as $\mathrm{L}$ Band, $S$ Band, $C$ Band, $X$ Band etc. To fulfil the demands of many users patch antenna is designed in these bands. Among different types of antenna, Microstrip Patch Antenna is most popular in wireless communication system. Microstrip patch Antennas have many advantages over other familiar antennas because microstrip patch antennas are of low profile, low cost, low volume, light weight. Low efficiency, low gain and narrow bandwidth of patch antenna create major challenge to a designer. Slots are created on patch for preparing antenna for wideband applications. In this paper, we have surveyed upon various types of Microstrip Patch Antenna, feeding techniques, design equation Substrate Characteristics, Simulation tools etc.
\end{abstract}

Keywords: Advantages, Feeding techniques, MPA, Patch antenna, Simulation.

\section{Introduction}

Antenna is designed in such a way that, it will transmit or receive electromagnetic waves that is antennas are of two types receiving and transmitting. Receiving antenna is kept at the place of most radiating place from transmitting antenna. Microstrip Patch Antennas (MPA) are printed directly on a circuit board. It is more familiar and suitable and essential for Mobile phone market, Satellite Communications, Radars, Aerospace etc. MPA has good return loss as well as Voltage Standing Wave Ratio (VSWR) and bandwidth (BW) [1]. MPA in its simplest form is shown in the figure 1.

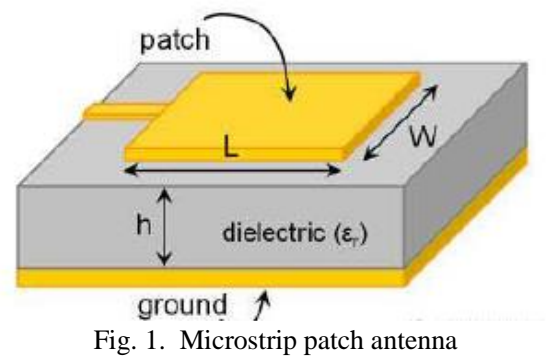

A dielectric substrate is situated between metallic patch and metallic ground. The dielectric constant of substrate lies between 2.2 and 12 [2]. All microstrip antennas can be divided into four basic categories (Figure 2).

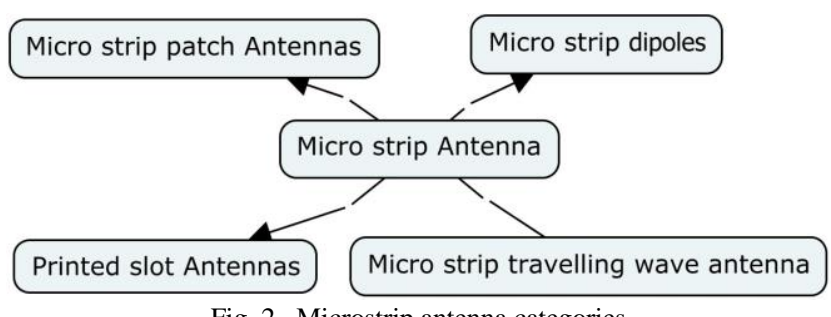

Fig. 2. Microstrip antenna categories

This paper is organized into following sections. In section 2 we discuss Microstrip patch antenna. This is followed by feeding techniques for antennas in section 3. Section 4 is devoted to equations for designing rectangular patch antenna. Section 5 describes effect of substrate characteristics on the design of Microstrip patch antenna. In section 6 we discuss on some of the standard simulation software in use for microstrip antennas.

\section{Microstrip Patch Antenna}

Microstrip patch antennas came in introduction in 1970 but in focus in 1950. Microstrip antenna (Figure 1) has a very thin $\left(\mathrm{t}<<\lambda_{0}\right.$, where $\lambda_{0}$ is the free-space wavelength) metallic strip(patch) and height of dielectric substrate $h$ and the value of $\mathrm{h}$ be $\left(\mathrm{h}<<\lambda_{0}\right.$, usually $\left.0.003 \lambda_{0}<\mathrm{h}<0.05 \lambda_{0}\right)$. For a rectangular patch, if $\mathrm{L}$ be length of the element then usually $\lambda_{0} / 3<\mathrm{L}<\lambda_{0} / 2$. Characteristics of dielectric substrate take important role in the antenna performance. If the substrate be thicker and dielectric constant be of lower, then antenna gives better efficiency and larger bandwidth but the size of antenna will be larger [4]. If we use substrate which is thin and of higher dielectric constant, then the element size will be small. This type of antenna is suitable to take challenge of modern communication [2].

The radiating patch may assume the shape of square, rectangular, circular, elliptical, triangular, thin strip(Dipole) or 
any other configuration (Figure 3).

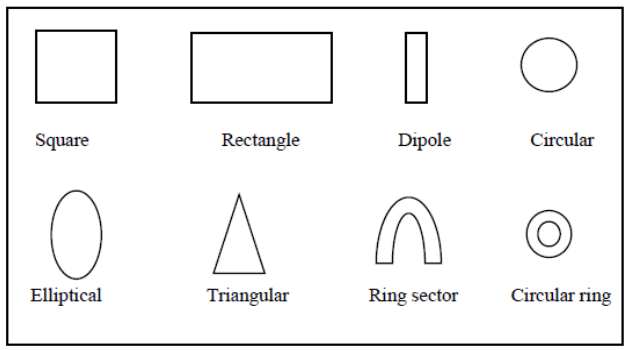

Fig. 3. Different shapes of patch antenna

Usually rectangular and circular size patches are used for microstrip antennas. The following chart will give clear idea about patch antennas at a glance. Profile of Microstrip patch antenna is thin, fabrication is easy, polarization is linear and circular both, spurious radiation exists and bandwidth is $2-50 \%$. These characteristics are important for getting information at a glance. Profile of microstrip slot antenna is thin, fabrication is easy, polarization is linear and circular both, spurious radiation also exists but bandwidth for such antenna is seen 5-30\% [3], [5].

\section{Feeding Techniques}

Feeding technique is very important for antenna operation. Through feed energy is supplied to the antenna. There are two types of feeding techniques, one is contacting and other is non contacting. Microstrip line and coaxial probe are contacting feeding technique but aperture coupling and proximity coupling feeding techniques are non-contacting type [6].

1) Microstrip line feed (Offset Microstrip)

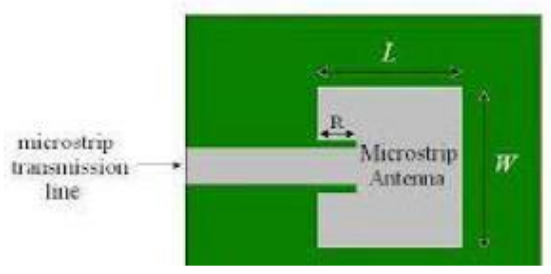

Fig. 4. Micro strip line feed

Here, a microstrip patch is directly connected with a conducting strip or microstrip line whose width is smaller than patch width (Figure 4). This technique is very easy and impedance matching by which is also simple.

2) Coaxial probe feed

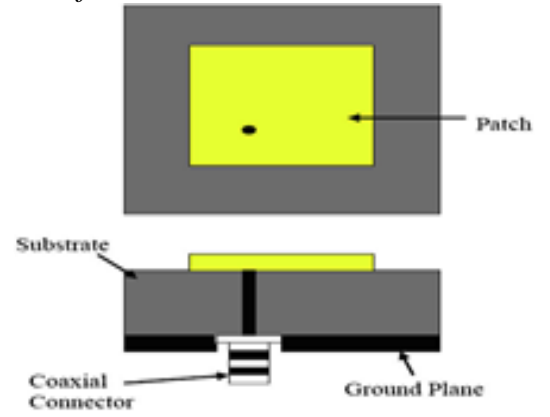

Fig. 5. Co-axial feed
Here feed be placed at any arbitrary location inside the patch for impedance matching. But narrow bandwidth is a disadvantage of this scheme. Here, a radiator patch is connected with the inner conductor of the co-axial. Ground is connected with outer conductor of co-axial.

\section{3) Aperture coupled feed}

Here, a ground plane separates two different substrates whose dielectric constants are chosen independently so that distinct electrical functions of circuit and radiations are optimized. On the bottom side of lower dielectric constant substrate there is a microstrip feed line. The feed energy is coupled to the patch through slot on the ground plane. Radiating patch is kept on the top position of upper substrate. Usually an antenna designer uses a high dielectric constant substrate for bottom substrate and a thick, low dielectric constant material for the top substrate for optimizing radiations from the patch.

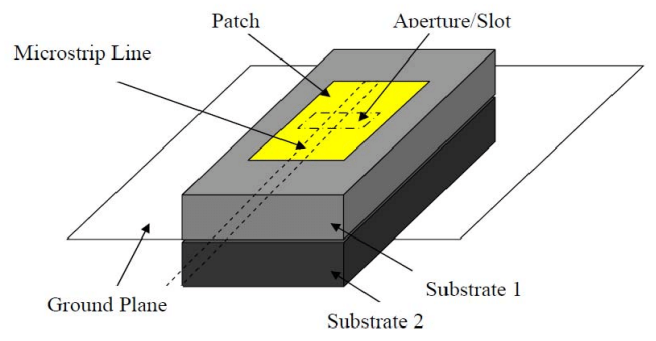

Fig. 6. Aperture coupled feed

\section{4) Proximity coupled feed}

Here we use two dielectric substrates whose proper alignment or array or make up is necessary. Radiating metal patch is placed on top of upper substrate. Feed line is attached between two substrates. By this technique we can eliminate false or spurious feed radiation. As the electrical thickness of microstrip patch antenna is enhanced through this technique so the large bandwidth of $13 \%$ is obtained. In this system electromagnetic waves are coupled so this feeding technique is called as electromagnetic coupling system or scheme [6]-[8].

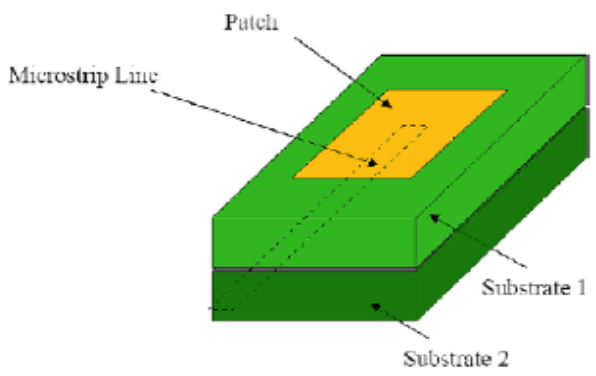

Fig. 7. Proximity coupled feed

The above feeding techniques are essential for designing an antenna. Because the study of different techniques will help a designer the knowledge about excitation and energy supply to the patch. Which technique can be used for better achievements in designing is also obtained from the above study.

Comparisons of different feeding techniques from the literature survey courtesy [10], [11]. 
Table 1

Comparisons of different feeding techniques

\begin{tabular}{|c|c|c|c|c|}
\hline Characteristics & Aperture coupled feed & Proximity coupled feed & Coaxial feed & Microstrip feed line \\
\hline Fabrication System & Alignment or array required & Array is required & Soldering and drilling machines are required & Easy \\
\hline Impedance matching & Easy & Easy & Easy & Easy \\
\hline Reliability & Good & Good & Poor & Bore \\
\hline Spurious feed radiation & Less & Minimum & $2-5 \%$ & More \\
\hline Bandwidth & $21 \%$ & $13 \%$ & $2-5 \%$. \\
\hline
\end{tabular}

In the above table feeding techniques are tabled in the descending band width order. For all techniques impedance matching is easy.

\section{Equations for Designing Rectangular Patch Antenna}

The design equations are described in below [2], [8], 12]. Notations used are frequency of operation $\left(f_{r}\right)$ dielectric constant of the substrate $\left(\epsilon_{r}\right)$ height of dielectric substrate $(h)$. Mainly these three parameters are essential for designing a patch antenna.

Width (W) of patch

$$
W=\frac{2}{f_{r}} \sqrt{\frac{2}{\varepsilon_{r}+1}}
$$

Calculation of effective dielectric constant $\left(\varepsilon_{r}^{\text {eff }}\right)$

Fringing effect has a role to calculate this.

$$
\varepsilon_{r}^{\text {eff }}=\frac{1}{2}\left(\varepsilon_{r}+1\right)+\frac{1}{2}\left(\varepsilon_{r}-1\right) 1+12\left(\frac{h}{W}\right)^{-1 / 2}
$$

Calculation of effective length $\left({ }^{L_{\text {eff }}}\right)$ of patch

$$
L_{e f f}=\frac{c}{2 \sqrt{\varepsilon_{r}^{e f f}}}\left(\frac{1}{f_{r}}\right)
$$

Calculation of length extension $(\Delta L)$. For designing a patch antenna, the extended patch length is considered. It comes due to fringing effect

$$
\Delta L=0.412 h\left[\frac{\left(\varepsilon_{r}^{\text {eff }}+0.3\right)\left(\frac{W}{h}+0.264\right)}{\left(\varepsilon_{r}^{\text {eff }}-0.258\right)\left(\frac{W}{h}+0.8\right)}\right]
$$

\section{Calculation of length of patch $(L)$}

Finally, the length of the patch of the antenna can be calculated by the following equation.

$$
L=L_{e f f}-2 \Delta L
$$

Calculation of ground plane dimensions

A designer can use the following equations for designing ground and substrate dimension.

$$
\begin{aligned}
& L_{g}=12 h+L \\
& W_{g}=12 h+W
\end{aligned}
$$

$h$ being the substrate height. $\mathrm{L}_{\mathrm{g}}$ and $\mathrm{W}_{\mathrm{g}}$ are length of ground and width of ground respectively.

Port and radiation box design: After feed point detection the ports are designed. Through port excitation is provided and the radiation box dimension must be larger than ground or substrate.

There are two types of port mainly lumped and wave ports take part in excitation.

\section{Advantages and disadvantages}

Microstrip patch antenna several advantages and disadvantages.

Advantages: Low weight, Low profile, Thin profile, Linear and circular polarizations, capable of dual and triple frequency operation, at a time feeding lines and matching networks can be designed.

Disadvantages: Insufficient efficiency, insufficient or low gain, low power handling capacity, excitation of surface waves, polarization impurity, large ohm loss in feeding structure.

\section{Effect of Substrate Characteristics on Microstrip Patch Antenna Design}

For designing an MPA, a designer should select the substrate material and its thickness with priority. Researcher should have clear idea about the effect of changing substrate material and its thickness on the performance of the antenna. We list some of the commonly used substrate materials and their dielectric constants which are equally useful for MPAs [11].

Thick substrate but low dielectric constant has some advantages and disadvantages in design process. Advantages are we get excellent efficiency, larger bandwidth and for radiation in space the loosely bound fields. After using such substrate, we see antenna element size becomes larger and weight, dielectric loss and surface wave loss of antenna increase also.

If we use thin substrate whose dielectric constant is high, then we see smaller size antenna. Because, this type of substrate demands tightly bound fields for minimizing unexpected

Table 2

\begin{tabular}{|l|l|l|l|l|l|l|l|}
\hline Materials & RT Duroid 5880 & GML 1000 & RO4003 & RF4 & $\mathrm{SiO}_{2}$ & Arlon AD430 & Arlon AD410 \\
\hline Dielectric constant & 2.2 & 3.2 & 3.4 & 4.4 & 4 & 4.3 & 4.1 \\
\hline
\end{tabular}


radiation and coupling. Disadvantage of this type antenna using such substrate is less efficiency and smaller bandwidth. For better performance the balance between efficiency and bandwidth which oppose each other. To bring this balance is a great challenge.

Higher dielectric constant material declines the antenna performance. Larger thickness of dielectric substrate reduces the size of antenna element and for such type of substrate resonance frequency decreases but at the cost of bandwidth. Higher dielectric constant material also decreases resonance frequency and increases bandwidth. These observations are obtained from different antenna simulation results and analysis.

\section{Simulation Software for Microstrip Antennas}

There are various simulation softwares by which we can design geometrical model, simulate, analysis the design, interpret the result and modify the design. There are HFSS, CST, IE3D, MATLAB simulation softwares.

HFSS: It is a commercial method solver for electromagnetic structures from Ansys. High Frequency Structure Simulator. General procedure for creating and analyzing a design is in the following.

First: Create a project for HFSS.

Second: Draw a geometrical model.

Third: Set up the problem.

Fourth: Generate the solution.

Fifth: Analyze the solution result.

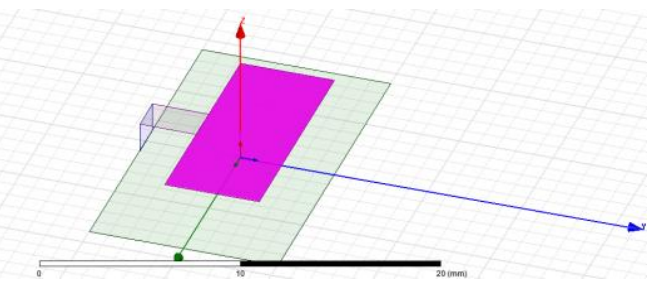

Fig. 8. Microstrip patch antenna designed in HFSS

This above microstrip patch antenna is designed in HFSS. Thus other software can be used in designing and analyzing various patch antenna also.

\section{Design of a MPA for Wideband Communication}

After implementing suitable feeding techniques, design equations, impedance matching a designer can think to design a MPA for wideband communication. During designing the designer should care on impedance bandwidth which is just an ordinary bandwidth of antenna. Impedance bandwidth is the range of frequencies over which the return loss is accepted. Cuts of different sizes or more than one slots are drawn on radiating patch of microstrip patch antenna to implement patch antenna for wideband applications. After designing MPA has to analyze. For this S Parameter, Z Parameter, Radiation patterns are plotted in any simulation software. From analyzing it will be seen that the frequency range where MPA is working. Thus a huge number of people will obtain MPA as gift for wideband communication system. Because wideband communication gives faster communication with high data rates.

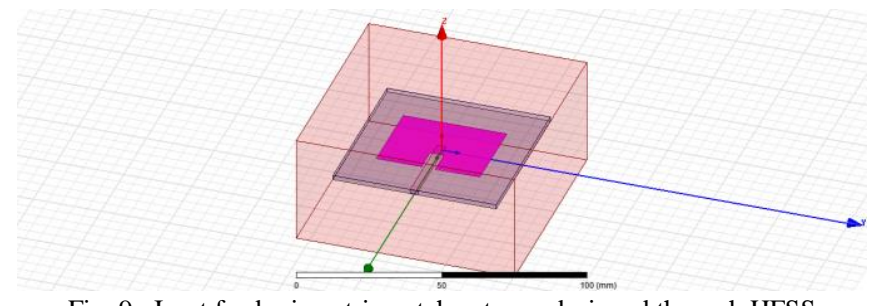

Fig. 9. Inset feed microstrip patch antenna designed through HFSS

It is an inset feed microstrip patch antenna which is designed through HFSS. If the slots are drawn on patch, then it will work in wide band application.

\section{Conclusion}

In this paper a theoretical survey on MPA has been done which addresses the feeding technique, design technique, advantage and disadvantages of MPA and the design techniques for designing MPA for wideband communication system. From analysis of this survey a designer can select best feeding techniques for minimizing the disadvantage. Also, a brief discussion is given on some of simulation software in use. MPA can be designed for each application and different merits have been compared with conventional microwave antenna. How dielectric constant of substrate affects the process is also explained here. This survey will help a designer in the designing process.

\section{References}

[1] V. P. Singh and M. Thakur, "Bandwidth Enhancement of Microstrip Line Inset Feed Patch Antenna," in IJERT, vol. 3. August 2014.

[2] A. Balanis, "Antenna Theory Analysis and Design," 3rd edition.

[3] K. Mamta, R. K. Singh, N. K. Sinha and R. K. Kesari, "Design and Development of Microstrip Patch Antenna for Millimeter Wave Application," Lecture Notes in Electrical Engg., 476, pp. 79-85, Springer Nature, July 2018.

[4] G. A. Deshamps, "Microstrip Microwave Antennas." Presented at the third USAF Symposium on Antennas, 1953.

[5] D. M. Pozar, "Microstrip Antenna," Proc, IEEE, vol. 80, no. 1, pp. 79-81, January 1992.

[6] N. Gupta, and D. Gangwar, "Survey on Microstrip Antenna," in International Journal of Engineering and Management Research, vol. 7, no. 2, pp. 189-193, March-April 2017.

[7] S. Bisht et al., "Study The Various Feeding Techniques of Microstrip Antenna Using Design and Simulation Using CST Microwave Studio," in International Journal of Emerging Technical and Advanced Engineering, vol. 4, no. 9, pp. 318-324, September 2014.

[8] K. Mamta and R. K. Singh, "Frequency Reconfigurable Millimeter Wave Antenna design for 5G Application," Int. J. of Mang., IT and Engg., vol. 9, no. 4, pp. 137-147, April 2019.

[9] I. J. Bahal and P. Bhatia, "Microstrip Antennas," Artech House, Dedham, M. A, 1980

[10] K. R. Carver and J. W. Mink, "Microstrip Antenna Technology," in IEEE Trans. Antennas propagant, vol. AP-29, no. 1, pp. 2-24, January 1981.

[11] K. Mamta and R. K. Singh, "Analysis of Reconfigurable Polarization Antenna as an EMI sensor," in British Journal of Applied Science and Technology, vol. 19, no. 5, pp. 1-8, March 2017. 
Volume-3, Issue-11, November-2020

https://www.ijresm.com | ISSN (Online): 2581-5792

[12] P. Singh et al., "Design and Simulation of W Shaped Microstrip Patch Antenna Using Different Feeding Techniques," in International Journal of Advanced Research in Electrical, Electronics and Instrumentation Engineering, vol. 2, no. 9, September 2013.

[13] V. P. Singh and Manoj Thakur, "Band width Enhancement of Microstrip Line Inset Feed Path Antenna," in IJERT, vol. 3, August 2014.
[14] I. Singh, and V. S. Tripathi, "Microstrip Patch Antenna and its Application: A survey," in Int. J. Comp. Tech. Appl., vol. 2, no. 5, pp. 1595-1599, September - October 2011.

[15] T. Kiran et al., "Design of Microstrip patch Antenna for 5G Applications," IOSR Journal of Electronics and Communication Engineering, vol. 13, no. 1, pp. 14-17, Jan.-Feb. 2018. 